\title{
Ciclo económico y performance de la combinación de las estrategias de diversificación de producto y expansión internacional
}

\author{
Luis Ángel Guerras-Martín ${ }^{1 a}$, José Ángel Zúñiga-Vicente ${ }^{\mathrm{a}}$, Diana Benito-Osorio ${ }^{\mathrm{a}}$ \\ y Alberto Colino ${ }^{\mathrm{b}}$
}

${ }^{a}$ Universidad Rey Juan Carlos, España

${ }^{b}$ Universidad Complutense de Madrid, España

doi: $10.20420 /$ eni.2021.422

\begin{abstract}
Resumen
En este trabajo se analiza el efecto conjunto de las estrategias de diversificación de producto y expansión internacional sobre los resultados empresariales teniendo en cuenta el ciclo económico. El marco de análisis es una muestra representativa de empresas manufactureras españolas en el período 1994-2014. En dicho período, la economía española experimentó una de las etapas de mayor crecimiento económico (1994-2008), seguida por una fase de profunda crisis (2009-2014). Nuestro estudio revela que ambas estrategias corporativas conducen a mejores resultados en la etapa de crecimiento cuando la expansión internacional se combina con niveles moderados de diversificación de producto (estrategia de diversión de producto relacionada). Por su parte, cuando las empresas optan por altos niveles de diversificación de producto (estrategia de diversificación de producto no relacionada), la combinación con la estrategia de expansión internacional solo lleva a mejores resultados en la etapa de crecimiento.
\end{abstract}

Palabras clave: diversificación de producto, expansión internacional, rentabilidad, ciclo económico, crecimiento, crisis.

Clasificación JEL: F23, L25, M16.

Agradecimiento: Los autores agradecen el apoyo financiero proporcionado por el Ministerio de Ciencia, Innovación y Universidades [proyecto RTI2018-097447-B-I00].

Fuente de referencia: Zúñiga-Vicente, J.Á., Benito-Osorio, D., Guerras-Martin, L.Á., \& Colino, A. (2019). The effects of international diversification on the link between product diversification and performance in a boom and bust cycle: Evidence from Spanish firms (1994-2014). Journal of International Management, 25(4), 100687.

\section{Introducción}

Dos de las decisiones de índole estratégica más importantes que deben tomar los directivos de una empresa tienen que ver con la diversificación o no de su cartera de productos y la internacionalización o diversificación geográfica. De hecho, la diversificación de producto y geográfica son consideradas dos estrategias corporativas claves para el desarrollo y crecimiento empresarial (Ansoff, 1965; Qian, 2002). La evidencia revela que en la mayoría de las empresas son dos decisiones que se toman de manera conjunta o simultánea. La literatura se ha referido a este fenómeno como "diversificación global" (ver Chang y otros, 2016; Denis y otros, 2002; Kim y otros, 1989).

Desde el trabajo de Kim et al. (1989) se ha asistido a un animado debate sobre si las mencionadas estrategias pueden considerarse como complementarias o no; es decir, si la adopción conjunta de ambas estrategias contribuye realmente a mejorar (o, por contra, a empeorar) los resultados empresariales. A pesar de ser un tópico que ha atraído una atención creciente en el ámbito de la estrategia corporativa (Guerras-Martín y otros, 2020) la investigación vigente no proporciona evidencia concluyente al respecto. Los trabajos de Zúñiga-Vicente y otros (2019) y Benito-Osorio y otros (2020) revisan los

${ }^{1}$ Autor de correspondencia: luisangel.guerras@urjc.es. 
principales estudios realizados sobre este tema en las tres últimas décadas.

Tras repasar la literatura, se advierte que, aunque los estudios existentes se han realizado en distintos países y momentos temporales diversos, prácticamente ninguno de ellos ha prestado atención al posible impacto del ciclo económico. Nuestro trabajo pretende cubrir este hueco en la literatura previa, por cuanto se centra en analizar empíricamente la incidencia que puede tener cada etapa del ciclo económico (en términos de 'crecimiento' y 'crisis') en la relación entre la combinación de ambas estrategias de diversificación y los resultados empresariales.

Examinar la cuestión propuesta es relevante porque, dado que cada fase del ciclo económico puede plantear la necesidad de afrontar y dar repuesta a retos distintos, los directivos pueden verse obligados a adoptar estrategias diferentes en cada etapa para lograr mejores resultados y, así, garantizar la supervivencia de las empresas. Nuestro estudio permite conocer en qué medida esto es realmente así o no. Esto es importante, ya que de los hallazgos obtenidos se derivan algunas ideas sobre cómo los directivos pueden hacer frente a una situación como la actual, de grave crisis económica ocasionada por la pandemia de la COVID-19, en términos de elección de la combinación de las estrategias corporativas más apropiadas.

\section{El ciclo económico y sus efectos sobre el performance de la combinación de las estrategias de diversificación de producto y geográfica}

Ciertamente, los directivos combinan diferentes tipos de estrategias corporativas para que las empresas que dirigen sean competitivas y sobrevivan. Esto es lo que sucede con las estrategias de diversificación de producto $\mathrm{y}$ geográfica. Son estrategias interdependientes porque precisan compromisos de inversión para aprovechar mejor los recursos y capacidades similares en los distintos mercados de productos y geográficos en los que compiten las empresas (Boehe \& Jiménez, 2018; Teece, 2014). A priori, una empresa que implementa una estrategia de diversificación de producto podría ver mejorados sus resultados si también se expande internacionalmente. Esto se debe a que la diversificación de productos puede facilitar el aprovechamiento de recursos y capacidades estratégicas y explotar economías de escala y/o alcance en diferentes mercados geográficos (países), al tiempo que se diversifican los riesgos del mercado (Geringer y otros, 2000; Kim y otros, 1989; Tallman \& Li, 1996).

No obstante, es obvio que tales decisiones estratégicas pueden estar condicionadas también por las oportunidades y/o amenazas que surgen del entorno macroeconómico. Así, en una fase de recesión económica es más probable que aumente la presión sobre las empresas para buscar nuevas oportunidades en los mercados internacionales y poder, de esta manera, contrarrestar la caída de la demanda de sus productos en el mercado interno (Hautz y otros, 2014). Ante una situación de crisis mundial, las empresas también se enfrentan a una creciente presión competitiva internacional, ya que es bastante probable que empresas de otros países, que se encuentran también en recesión, exploren nuevas oportunidades fuera de sus mercados nacionales.

Por contra, en un período de crecimiento es más fácil encontrar oportunidades para diversificar la cartera de productos en el mercado nacional, enfrentándose al mismo tiempo a una menor presión de los rivales. Esto también podría reducir la necesidad de buscar nuevas oportunidades en otros países (Hautz y otros, 2014; Lim y otros, 2009). La expansión a otros países puede ayudar a las empresas que persiguen altos niveles de diversificación de producto (estrategia de diversificación de producto no relacionada) a lograr economías de escala, que a menudo no se pueden conseguir 
compitiendo exclusivamente en los mercados nacionales. Asimismo, puede permitir explotar otro tipo de sinergias, más allá de las estrictamente financieras, como son las tecnológicas, comerciales y/o directivas (Robins \& Wiersema, 1995; Zhang y otros, 2019).

Con todo, competir en diversos países también puede tener un efecto negativo en la capacidad de supervisión o vigilancia por parte de los directivos. Esto puede representar un serio desafío durante una fase de recesión. En este contexto, los directivos probablemente tienen menos capacidad para encontrar nuevas oportunidades que involucren simultáneamente diferentes productos y mercados geográficos debido al mayor nivel de incertidumbre (Bowen \& Sleuwaegen, 2017; Garrido-Prada y otros, 2018). La implementación de mecanismos de control interno efectivos también es más difícil y costosa (Chang \& Wang, 2007), por lo que los costes organizativos para las empresas que compiten en segmentos de productos diferentes y se expanden a otros países serán mayores. Por consiguiente, la combinación de las estrategias de diversificación de producto no relacionada y expansión internacional solo tendrá un efecto positivo sobre los resultados empresariales en una fase de crecimiento.

En cambio, para las empresas que optan por niveles moderados en la diversificación de producto (estrategia de diversificación relacionada), la oportunidad de lograr economías de escala y alcance, compartir recursos y competencias básicas, o explotar las interdependencias entre las diferentes unidades de negocio (o productos) puede aumentar notablemente cuando se expanden a otros mercados geográficos (Hitt y otros, 1994; Tallman \& Li, 1996). Estas empresas suelen tener menos problemas ocasionados por la gestión de productos diversos $\mathrm{y}$, por ende, les resulta menos costoso implementar controles estratégicos y financieros, pudiendo concentrarse en integrar los objetivos estratégicos de todas las unidades de negocio más eficazmente (Baysinger \& Hoskisson, 1989; Chang \& Wang, 2007).

Si las empresas diversifican su cartera de productos de forma relacionada se enfrentan a entornos competitivos, sistemas de distribución y preferencias de los clientes más similares. No necesitan dedicar tanto tiempo y recursos a realizar ajustes en todas las unidades de negocio, lo que reduce sustancialmente los costes organizativos (Chang \& Wang, 2007). De este modo, en una fase de crecimiento las empresas que adoptan una estrategia de diversificación de producto relacionada y se expanden internacionalmente pueden lograr más fácilmente todo el potencial de dicha interacción.

$\mathrm{Al}$ ser mayor la incertidumbre en un contexto de recesión, las empresas pueden necesitar una comunicación interna más intensa y continua para explotar los beneficios potenciales de la relación entre las distintas unidades de negocio. Estas relaciones de cooperación podrían llevar a una pérdida de autonomía e independencia para los responsables de las mismas, lo que podría redundar en respuestas más rígidas y lentas a la demanda en mercados nacionales específicos (Chang \& Wang, 2007; Qian, 1997). Los costes de gobierno asociados a estos problemas podrían reducir la contribución positiva de la expansión internacional sobre los resultados en las empresas que adoptan una estrategia de diversificación de producto relacionada, aunque seguirán siendo positivos. Por tanto, la combinación de una estrategia de diversificación de producto relacionada con la expansión internacional tendrá un efecto positivo sobre los resultados empresariales tanto en una fase de crecimiento como de recesión, aunque en el último caso el efecto positivo será menor.

\section{Metodología y resultados}

Se utiliza una muestra de empresas manufactureras españolas de la Encuesta de Estrategias Empresariales (ESEE) entre 1994 y 
2014; en concreto, de un panel de 2.565 empresas y un total de 23.063 observaciones tras realizar los procesos de depuración de datos pertinentes. El período analizado comprende dos etapas muy diferentes del ciclo económico en España: 'crecimiento' (1994-2008) y 'crisis' (20092014).

El período 1994-2008 coincide con una de las fases de crecimiento más largas de la economía española en los últimos cincuenta años. Entre 1994 y 2008, la tasa de crecimiento acumulada del PIB fue del 54\%, lo que significa un crecimiento medio anual del 3,6\%, mientras que el PIB per cápita pasó de $10.800 €$ en 1994 a $24.300 €$ en 2008. España llegó a ser la octava potencia económica del mundo.

En el período 2009-2014 se sufrió una de las peores crisis económicas, hasta la actual pandemia de la COVID-19. La crisis se hizo patente en España en 2009, y sus efectos fueron devastadores: implicó una fuerte caída del PIB, un aumento drástico del desempleo y un gran número de quiebras de empresas en la mayoría de los sectores económicos. Por ejemplo, el PIB cayó un 9.2\%. El PIB per cápita en 2014 fue de $22.780 €$. La tasa de desempleo aumentó del $13,8 \%$ en 2008 al $23,7 \%$ en 2014. El número total de empresas entre 2008 y 2014 disminuyó alrededor de un 9\%.

Los principales hallazgos se resumen gráficamente en las Figuras 1 y 2. En el eje vertical de ambas figuras se muestra la variable de resultados empresariales considerada - Rentabilidad Económica (RE). El eje horizontal recoge el grado de diversificación de producto (DP) — medido a través del índice de entropía. Un valor de 0 indica que la empresa no está diversificada, valores bajos-moderados se asimilan a una estrategia de diversificación relacionada, y valores elevados a una estrategia de diversificación no relacionada. La línea continua muestra la relación entre los resultados (RE) y la estrategia de diversificación de producto. La línea discontinua tiene en cuenta cómo es la relación de ambos tipos de estrategias corporativas consideradas conjuntamente, sobre los resultados empresariales (RE). $\mathrm{La}$ diversificación geográfica (DG) se mide mediante el cociente entre las ventas en el extranjero y las ventas totales de la empresa.

La Figura 1 muestra que la combinación de las estrategias de diversificación de producto $\mathrm{y}$ de expansión internacional (DP * DG) suponen una mejora en los resultados (RE) en comparación con las empresas que solo siguen una estrategia de diversificación de producto (DP) en la fase de crecimiento. Esta figura también refleja que los resultados más elevados se alcanzan cuando se combinan la estrategia de expansión internacional con altos niveles de diversificación de producto (estrategia de diversificación de producto no relacionada), aunque la combinación con moderados niveles de diversificación también conlleva un aumento de la rentabilidad.

Figura 1. Relación entre la diversificación de producto y geográfica sobre los resultados en la fase de 'crecimiento'

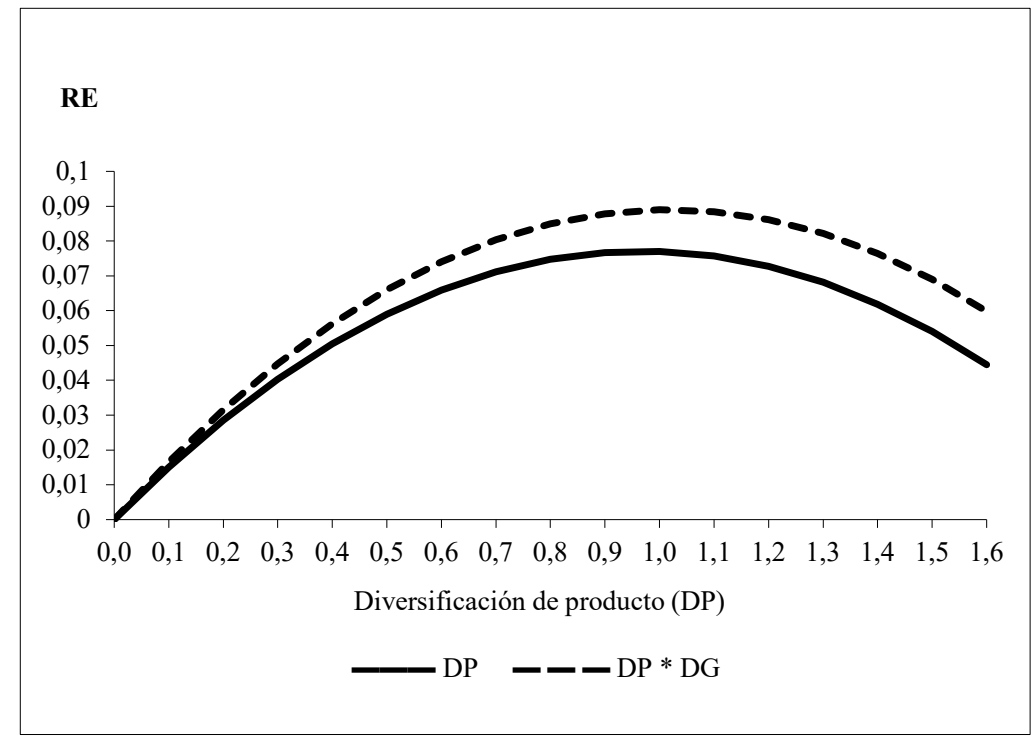

Fuente: Zúñiga-Vicente et al. (2019,p. 16).

La Figura 2 revela que la combinación de ambos tipos de estrategias corporativas (DP* DG) solo supone una mejora en los resultados 
con respecto a las empresas que siguen una estrategia de diversificación de producto cuando tales empresas optan por niveles bajosmoderados de diversificación de producto (estrategia de diversificación relacionada) en la fase de crisis. Cuando en dicha fase se combina la estrategia de expansión internacional con altos niveles de diversificación de producto (estrategia de diversificación no relacionada) los resultados empresariales empeoran.

Figura 2. Relación entre la diversificación de producto y geográfica sobre los resultados en fase de 'crisis'

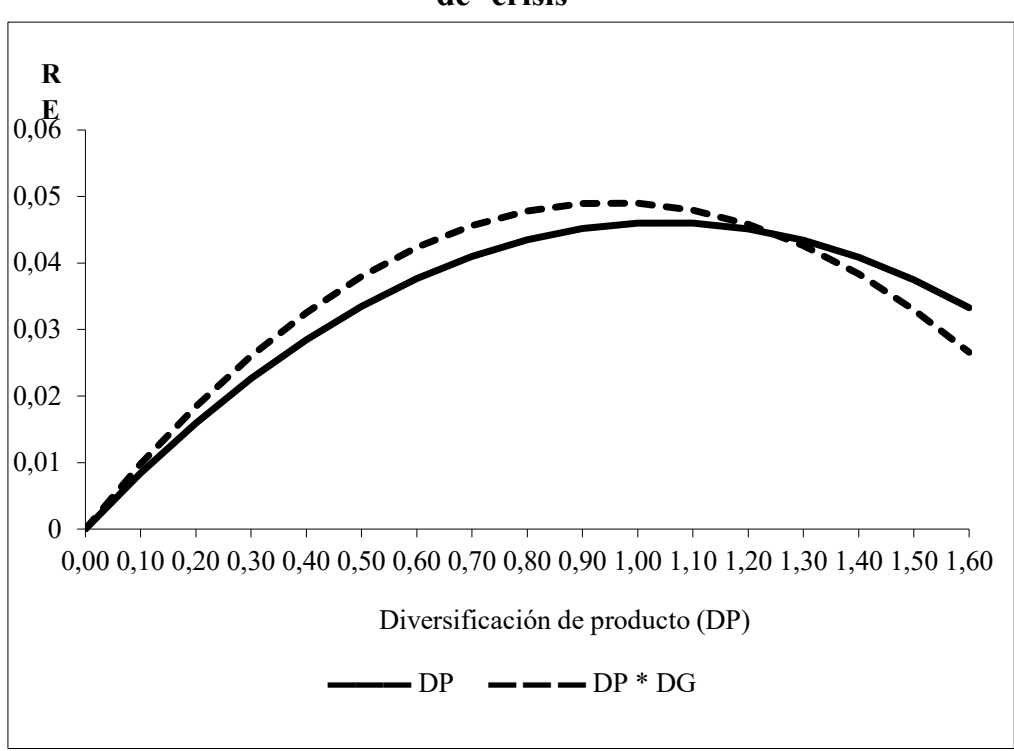

Fuente: Zúñiga-Vicente et al. (2019, p. 16)

\section{Conclusiones e implicaciones prácticas}

Podemos concluir que los resultados de las empresas que combinan las estrategias de diversificación de producto $\mathrm{y}$ expansión internacional dependen del ciclo económico. En concreto, ambas estrategias son complementarias en una etapa de crecimiento, ya que su efecto combinado sobre la rentabilidad es positivo. En esta etapa, la expansión internacional ayuda a mejorar la rentabilidad de las compañías con niveles bajos, moderados $y$ altos de diversificación de producto (empresas con estrategias de diversificación tanto relacionada como no relacionada). Por su parte, en una fase de crisis, ambas estrategias corporativas pueden ser complementarias o sustitutivas; la expansión internacional mejora la rentabilidad de las compañías con niveles bajos-moderados de diversificación de producto, pero la empeora cuando la estrategia de expansión internacional va acompañada de altos niveles de diversificación de producto.

Nuestros hallazgos sugieren que los directivos de las empresas manufactureras deberían optar por estrategias de diversificación de producto a un nivel moderado-alto (diversificación relacionada o no relacionada) para lograr altas tasas de rentabilidad en fases de crecimiento, pero solo a un nivel moderado en períodos de crisis. Con todo, han de ser conscientes de que existe un umbral o valor óptimo para la diversificación de producto en ambas fases. Este umbral puede diferir para cada empresa en función de su dotación de recursos y capacidades. Dado que las estrategias de diversificación de producto y expansión internacional son complementarias en la fase de crecimiento, para mejorar la rentabilidad, los directivos podrían aumentar dicho umbral de diversificación de producto si también optan por la expansión a nuevos mercados geográficos.

Por otro lado, deberían apostar por un nivel bajo-moderado de diversificación de producto si también optan por una expansión internacional durante una recesión económica si desean obtener el máximo potencial de ambas estrategias, en términos de rentabilidad. Extrapolando estos hallazgos a la situación de la crisis económica actual, causada por la COVID19 , nuestra recomendación es que los directivos de las empresas manufactureras españolas opten simultáneamente por niveles moderados de diversificación de producto (estrategia de diversificación relacionada) y una estrategia de expansión internacional; en vista de la experiencia previa, esta parece ser la combinación de estrategias corporativas más 
adecuada para afrontar de manera satisfactoria un contexto de crisis.

\section{Referencias}

Ansoff, H.I. (1965). Corporate Strategy: An Analytic Approach to Business Policy for Growth and Expansion. New York: McGraw-Hill.

Baysinger, B., \& Hoskisson, R.E. (1989). Diversification strategy and R\&D intensity in multiproduct firms. Academy of Management Journal, 32(2), 310-332.

Benito-Osorio, D., Colino, A., Guerras-Martín, L.Á., \& Zúñiga-Vicente, J.Á. (2020). The combined effects of product and geographical diversification on performance: Evidence in manufacturing SMEs. $B R Q$ Business Research Quarterly, 23(2), 91-106.

Boehe, D., \& Jiménez, A. (2018). Does the sequencing of related and unrelated export diversification matter? Evidence from Colombian exporters. International Business Review,27(6), 1141-1149.

Bowen, H.P., \& Sleuwaegen, L. (2017). Are international and product diversification substitutes or complements? Theoretical and empirical perspectives. Global Strategy Journal, 7(3), 241-256.

Chang, S., Kogut, B., \& Yang, J-S. (2016). Global diversification discount and its discontents: A bit of self-selection makes a world of difference. Strategic Management Journal, 37(11), 2254-2274.

Denis, D.J., Denis, D.K., \& Yost, K. (2002). Global diversification, industrial diversification, and firm value. The Journal of Finance, 57(5), 1951-1979.

Garrido-Prada, P., Delgado-Rodriguez, M.J., \& Romero-Jordán, D. (2019). Effect of product and geographic diversification on company performance: Evidence during an economic crisis. European Management Journal, 37(3), 269-286.

Geringer, J.M., Tallman, S., \& Olsen, D.M. (2000). Product and international diversification among Japanese multinational firms. Strategic Management Journal, 21(1), 51-80.

Guerras-Martín, L.Á., Ronda-Pupo, G.A., ZúñigaVicente, J.Á., \& Benito-Osorio, D. (2020). Half a century of research on corporate diversification: A new comprehensive framework. Journal of Business Research, 114, 124-141.

Hautz, J., Mayer, M., \& Stadler, C. (2014). Macrocompetitive context and diversification: the impact of macroeconomic growth and foreign competition. Long Range Planning, 47(6), 337-352.

Hitt, M.A., Hoskisson, R.E., \& Ireland, R.D. (1994). A mid-range theory of the interactive effects of international and product diversification on innovation and performance. Journal of Management, 20(2), 297-326.

Kim, W.C., Hwang, P., \& Burgers, W.P. (1989). Global diversification strategy and corporate profit performance. Strategic Management Journal, 10(1), 45-57.

Lim, E. N-K, Das, S.S., \& Das, A. (2009). Diversification strategy, capital structure, and the Asian financial crisis (1997-1998): Evidence from Singapore firms. Strategic Management Journal, 30(6), 577-594.

Qian, G. (1997). Assessing product-market diversification of US firms. Management International Review, 37(2), 127-149.

Qian, G. (2002). Multinationality, product diversification, and profitability of emerging US small- and medium-sized enterprises. Journal of Business Venturing, 17(6), 611-633.

Robins, J., \& Wiersema, M.F. (1995). A resourcebased approach to the multibusiness firm: Empirical analysis of portfolio interrelationships and corporate financial performance. Strategic Management Journal, 16(4), 277-299.

Tallman, S., \& Li, J.T. (1996). The effects of international diversity and product diversity on the performance of multinational firms. Academy of Management Journal, 39(1), 179-196.

Teece, D.J. (2014). A dynamic capabilities-based entrepreneurial theory of the multinational enterprise. Journal of International Business Studies, 45(1), 8-37.

Zhang, F., Jiang, G., \& Cantwell, J. A. (2019). Geographically dispersed technological capability building and $\mathrm{MNC}$ innovative performance: The role of intra-firm flows of newly absorbed 
knowledge. Journal of International international diversification on the link between Management, 25(3), 100669. product diversification and performance in a boom

Zúñiga-Vicente, J.Á., Benito-Osorio, D., GuerrasMartín, L.Á., \& Colino, A. (2019). The effects of and bust cycle: Evidence from Spanish firms (19942014). Journal of International Management, 25(4), 100687. 